\title{
Dust obscuration of DLA galaxies
}

\author{
Giovanni Vladilo ${ }^{1}$ \\ ${ }^{1}$ Istituto Nazionale di Astrofisica, Osservatorio Astronomico di Trieste, Trieste, Italy \\ email: vladilo@ts.astro.it
}

\begin{abstract}
We show that the extinction of quasar absorbers increases exponentially with the logarithmic column density of any volatile metal (e.g. zinc), with a characteristic turning point above which the quasars are severely dimmed. We derive the relation between extinction, HI column density $\left(N_{\mathrm{HI}}\right)$, metallicity $\left(Z \equiv N_{\mathrm{ZnII}} / N_{\mathrm{HI}}\right)$, and fraction of iron in dust $\left[f_{\mathrm{Fe}}(Z)\right]$ in Damped Lyman $\alpha$ (DLA) systems. We use this relation to estimate the effect of dust obscuration on the statistical distributions of $N_{\mathrm{HI}}$ and $Z$ measured in magnitude-limited surveys of DLAs. In the redshift range where the measurements of zinc column densities have sufficient statistics $(1.8 \leqslant z \leqslant 3)$ we find that the obscuration bias affects the shapes of the observed distributions. The metallicity distribution is particularly affected by the bias, which hampers the detection of DLA galaxies with near Solar metallicity. Our results perfectly reproduce, without tuning the dust parameters, the DLA observational threshold $\log N(\mathrm{ZnII})\left[\mathrm{cm}^{-2}\right] \lesssim 13.15$ found by Boissé and collaborators in 1998, in terms of a rapid rise of the obscuration. Our predictions of the effects of the bias on the statistics of DLAs are consistent with observational results obtained from unbiased surveys of radio-selected quasars.
\end{abstract}

\section{Introduction}

A variety of astronomical observations indicate that interstellar dust is a pervasive galactic component, not only in the Milky Way and nearby galaxies, but also in the high-redshift Universe (Meurer 2004). Among quasar absorbers, damped Lyman $\alpha$ (DLA) systems are the best candidates to host dust because of their high HI column density, which shields the grain from ionising photons, and because of their metal content, which is higher than in other absorbers. If dust is present in DLA systems, it will scatter and absorb the radiation of the background quasar, dimming its apparent magnitude (extinction) and changing the slope of its energy distribution (reddening). In the most extreme cases, the extinction may obscure the quasar leading to a selection effect, the obscuration bias, which may prevent the detection of metal-rich, dusty DLAs (Ostriker \& Heisler 1984; Fall \& Pei 1993).

Measuring the extinction or reddening of DLAs is a difficult task owing to the uncertain, variable continuum of the quasars. Detection of quasar reddening from intervening DLAs was first claimed by Pei et al. (1991). Recent investigations based on large data sets find reddening from metal absorbers at redshift $1 \leqslant z \leqslant 1.9$ (Khare et al. 2005), but not from DLAs at $z \sim 2.8$ (Murphy \& Liske 2004). Differential reddening in two lines of sight of one DLA at $z=0.9$ has been reported from the study of a gravitationally lensed quasar (Lopez 2005).

Evidence of the obscuration bias has been searched by comparing the statistics of magnitude-limited surveys with those of complete surveys of DLAs lying in front of radioselected quasars. The CORALS survey has provided a marginal signal of obscuration in the absorber number density, the neutral gas content (Ellison et al. 2001) and the metallicity (Ellison 2005), but only at $\approx 1 \sigma$ level in each case. 
The lack of DLA systems with high values of metallicity and HI column density, above the threshold $\log N(\mathrm{ZnII})\left[\mathrm{cm}^{-2}\right] \simeq 13.15$, was tentatively attributed to the effect of dust obscuration (Boissé et al. 1998). The original motivation of the present work was to understand if that threshold can be quantitatively explained in terms of a fast rise of the DLA extinction, in which case it would represent an important clue to the obscuration effect. With this goal in mind, we first derived the relation between interstellar extinction and $N(\mathrm{ZnII})$. We then used this relation to quantify the effect of the bias on the distributions of column densities and metallicities of DLAs. Finally, we applied our method to the current sample DLAs with ZnII measurements. These three parts of our work are briefly outlined in the next three sections. More details can be found in the full paper (Vladilo \& Péroux 2005).

\section{The extinction law}

The extinction in a photometric band with effective wavelength $\lambda, A_{\lambda}$, is related to the line-of-sight zinc column density, $N_{\mathrm{Zn}} \equiv N(\mathrm{ZnII})$, by means of the relation

$$
A_{\lambda}=a_{\lambda} 10^{\log N_{\mathrm{Zn}}}
$$

where

$$
a_{\lambda}=1.817 \times 10^{-24} \mathrm{~A}_{\mathrm{Fe}} \times g_{\lambda} \times \frac{f_{\mathrm{Fe}}}{\left(1-f_{\mathrm{Zn}}\right)} \times\left(\frac{\mathrm{Fe}}{\mathrm{Zn}}\right) .
$$

These relations can be derived by comparing the column densities of a refractory and a volatile element in a given line of sight (we adopted iron and zinc, respectively). The expression (2.2) is composed of: (1) constant factors (e.g. $A_{\mathrm{Fe}}$ is the atomic mass of iron); (2) a dust grains parameter, $g_{\lambda} ;(3)$ the fraction of iron and zinc atoms which are incorporated in the dust, $f_{\mathrm{Fe}}$ and $f_{\mathrm{Zn}}$; and (4) the relative abundance of iron and zinc in the medium, $(\mathrm{Fe} / \mathrm{Zn})$. This last term is expected to be constant, close to the Solar value, in the local ISM. The term $f_{\mathrm{Fe}} /\left(1-f_{\mathrm{Zn}}\right)$ is approximately constant and $\approx 1$ by the definition of refractory and volatile elements. The only factor expected to vary is

$$
g_{\lambda}=s \frac{Q_{e, \lambda}}{r_{\mathrm{gr}} \varrho_{\mathrm{gr}} X_{\mathrm{Fe}}^{\mathrm{d}}},
$$

where $s$ is a geometrical factor ( $=3 / 4$ for spherical grains), $Q_{e, \lambda}$ the extinction efficiency factor (Spitzer 1978), $r_{\mathrm{gr}}$ the grain radius, $\varrho_{\mathrm{gr}}$ the grain density, and $X_{\mathrm{Fe}}^{\mathrm{d}}$ the abundance by mass of iron in the dust.

We tested and calibrated the relation (2.1) using interstellar measurements of extinction and $N(\mathrm{ZnII})$. The results are shown in Fig. 1. The data follow the expected trend and yield a mean value of the $a_{\lambda}$ parameter in the $\mathrm{V}$ band $a_{V}=(0.29 \pm 0.07) \times 10^{-13} \mathrm{mag}$ nitudes $\mathrm{cm}^{-2}$. From the condition $\partial A_{\lambda} / \partial\left(\log N_{\mathrm{Zn}}\right)=1$ magnitude, we obtain a turning point $\log N(\mathrm{ZnII}) \simeq 13.18$, above which the extinction experiences a dramatic rise. This extinction barrier lies remarkably close to the Boissé threshold and this fact motivated us to study the extinction relation for DLAs. As a result, we obtained that the observer's frame extinction of a DLA at redshift $z$ is

$$
A_{\lambda}\left(N_{\mathrm{H}}, Z ; z\right) \simeq A_{\circ} G \xi\left(\frac{\lambda}{1+z}\right) f_{\mathrm{Fe}}(Z) N_{\mathrm{H}} Z,
$$

where $A_{\circ} \simeq 1.85 \times 10^{-14} \mathrm{mag} \mathrm{cm}{ }^{2}, G=\left(g_{\lambda}\right)_{\mathrm{DLA}} /\left(g_{\lambda}\right)_{\mathrm{ISM}}$, and $\xi(\lambda)=A(\lambda) / A\left(\lambda_{V}\right)$ is a normalised extinction curve; $Z=\mathrm{Zn} / \mathrm{H}$ and $N_{\mathrm{H}}$ are the metallicity and HI column density of the absorber; and $f_{\mathrm{Fe}}(Z)$ is an analytical function, based on depletion data (Vladilo 2004), that gives the fraction of iron in dust as a function of the metallicity. 

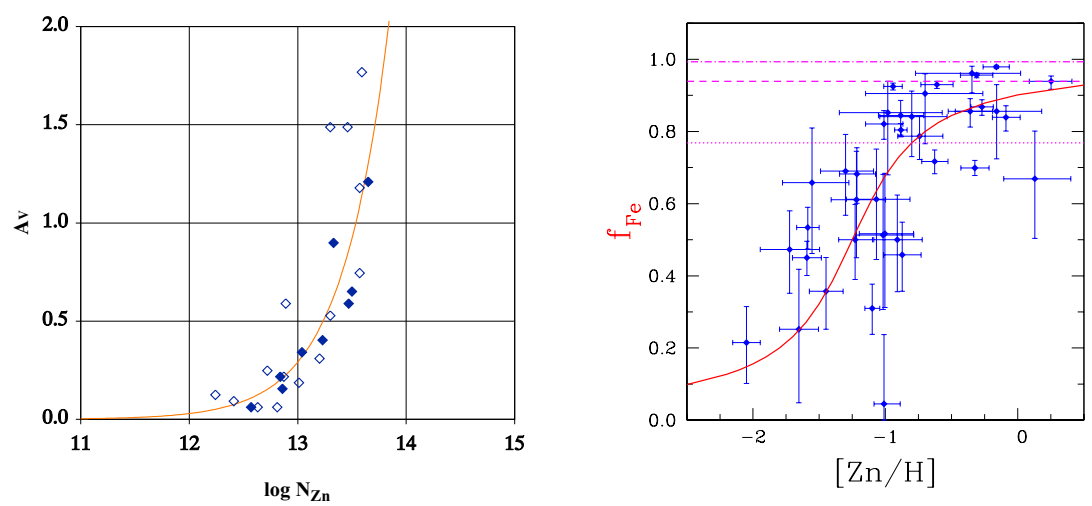

Figure 1. Left panel: $\mathrm{V}$ band extinction versus zinc column density in the interstellar medium (see Vladilo \& Péroux 2005). Right panel: fraction of iron in dust versus metallicity in DLAs (Vladilo 2004); smooth line: analytical approximation adopted in the present study.

Since $f_{\mathrm{Fe}}(Z)$ vanishes at low metallicities (Fig. 1, right panel), the predicted extinction will accordingly vanish. Thanks to this fact, we do not need to know the value of the grain parameter at the very early stages of chemical evolution, when the behaviour of the dust is most unpredictable. In the application of Eq. 2.4 we consider both a 'Milky-Way' and a Small Magellanic Cloud ('SMC') type of dust. For each type we adopt an appropriate $\xi(\lambda)$ and $G$, making use also of SMC extinction data (see details in the full paper).

\section{Method}

At a given redshift, the distribution of DLA extinctions and the obscuration bias are completely determined from Eq. 2.4 for any assigned pair of $N(\mathrm{HI})$ and $Z$ distributions. We used this property to find the relation between true and biased distributions of $N(\mathrm{HI})$ and $Z$ in DLAs. Simple mathematical expressions can be derived by assuming that the distributions of $N(\mathrm{HI})$ and $Z$ are statistically independent and by ignoring multiple DLA absorptions in a given line of sight. Both assumptions are conservative in the sense that they may slightly underestimate the effect of quasar obscuration. The fraction of DLAquasar pairs that can be detected in the differential element $d N_{\mathrm{H}} d Z d z$ is

$$
B_{m_{\ell}}\left(N_{\mathrm{H}}, Z, z\right) \equiv \frac{\int_{\circ}^{m_{\ell}-A_{\lambda}\left(N_{\mathrm{H}}, Z, z\right)} n(m ; z) d m}{\int_{\circ}^{m_{\ell}} n(m ; z) d m},
$$

where $A_{\lambda}\left(N_{\mathrm{H}}, Z, z\right)$ is the extinction (Eq. 2.4), $n(m)$ is the distribution of the quasar apparent magnitudes $m$, and $m_{\ell}$ the limiting magnitude of the survey. In the redshift interval $z_{1} \leqslant \bar{z} \leqslant z_{2}$ the relations between the true distributions of $N(\mathrm{HI})$ and $Z$ in DLAs, $f_{N_{\mathrm{H}}}$ and $f_{Z}$, and the corresponding biased distributions $f_{N_{\mathrm{H}}}^{\mathrm{b}}$ and $f_{Z}^{\mathrm{b}}$, are

$$
\begin{gathered}
f_{N_{\mathrm{H}}}^{\mathrm{b}} \simeq \frac{\int_{0}^{\infty} \mathcal{B}_{m_{\ell}}\left(N_{\mathrm{H}}, Z\right) f_{Z} d Z}{\int_{0}^{\infty} f_{Z} d Z} f_{N_{\mathrm{H}}}, \\
f_{Z}^{\mathrm{b}} \simeq \frac{\int_{N_{\mathrm{DLA}}}^{\infty} \mathcal{B}_{m_{\ell}}\left(N_{\mathrm{H}}, Z\right) f_{N_{\mathrm{H}}} d N_{\mathrm{H}}}{\int_{N_{\mathrm{DLA}}}^{\infty} f_{N_{\mathrm{H}}} d N_{\mathrm{H}}} f_{Z},
\end{gathered}
$$

where $\mathcal{B}_{m_{\ell}}\left(N_{\mathrm{H}}, Z\right)=B_{m_{\ell}}\left(N_{\mathrm{H}}, Z, \bar{z}\right)$ and $N_{\text {DLA }}=10^{20.3}$ atoms $\mathrm{cm}^{-2}$. The goal of our procedure is to invert these equations in order to recover the true distributions, given 


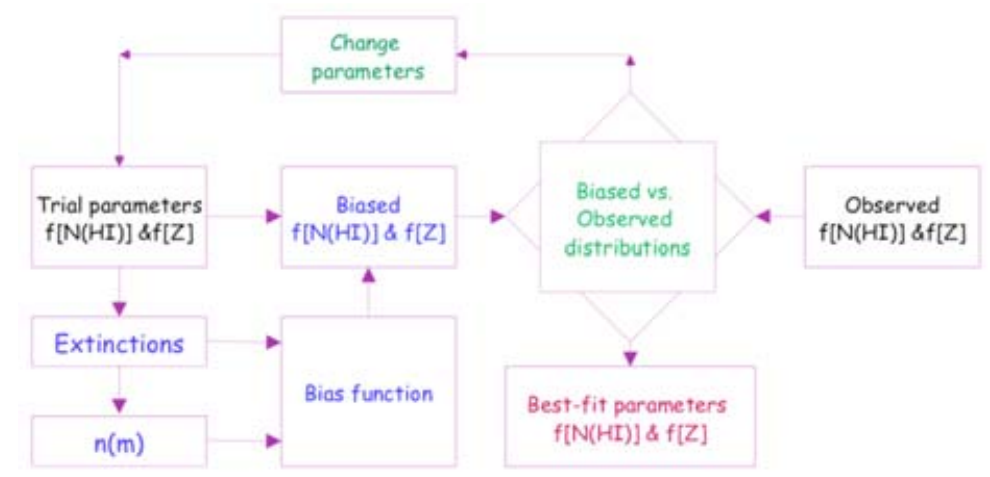

Figure 2. Flow diagram of the procedure adopted with the aim of recovering the true distributions of HI column densities and metallicities in DLAs starting from the observed distributions.

the biased ones, which can be estimated from magnitude-limited surveys. To do so we use the procedure sketched in Fig. 2. We model the unknown distributions $f_{N_{\mathrm{H}}}$ and $f_{Z}$ using analytical functions with free parameters. We start from a set of trial parameters and compute the extinction distribution, the function $\mathcal{B}_{m_{\ell}}\left(N_{\mathrm{H}}, Z\right)$ and the biased distributions. We then compute the $\chi^{2}$ deviation between the biased distributions and the observed ones. The procedure is iterated to find a minimum in the $\chi^{2}$ values. At each iteration we estimate the true distribution of quasar apparent magnitudes, $n(m)$, by correcting the observed magnitude distribution taking into account the effect of the extinction. In this way we do not need to assume a quasar luminosity function. For this and other reasons our procedure is different from the one presented by Fall \& Pei (1993).

\section{Results}

As a first application of our method we considered the sample of DLAs with ZnII data. The distribution of apparent magnitudes was measured from the quasar counts in the $r$ and $g$ bands of the Sloan Digital Sky Survey. The extinctions were estimated in the same two bands. The selected sample is characterised by a quasar limiting magnitude $m_{\ell} \simeq 19$ in the optical, required to detect the $\mathrm{ZnII}$ lines at high spectral resolution. Most data are concentrated in the range $1.8 \leqslant z \leqslant 3.0$, with a median redshift $z \approx 2.3$. The current limitation of the statistics (about 40 DLAs in 2004) allowed us to compute the empirical distributions in a small number of bins. Because of the limited empirical constraints we modelled the true distributions $f_{N_{\mathrm{H}}}$ and $f_{Z}$ using functions with the smallest possible number of parameters. For the $N(\mathrm{HI})$ distribution we adopted a power law $f_{N_{\mathrm{H}}}=C \times N_{\mathrm{H}}^{-\beta}$ truncated at the highest observed values of DLA column density, $\log N(\mathrm{HI}) \simeq 22$. For the metallicity distribution we adopted a Schechter function $f_{Z}=$ $C^{\prime} \times\left(Z / Z_{*}\right)^{\alpha} e^{-Z / Z_{*}}$. With tighter observational constraints available in the future, it will be possible to model the true distributions more realistically.

For the different types of dust (MW- and SMC-type) and photometric bands considered, we found that the slope of the $N(\mathrm{HI})$ power law lies in the range $1.5 \lesssim \beta \lesssim 1.6$, with typical fit errors of \pm 0.12 . The mean value $\langle Z>$ of the Schechter metallicity distribution lies in the range $-0.4 \lesssim \log \left(<Z>/ Z_{\odot}\right) \lesssim-0.3$, with fit errors of \pm 0.15 dex and total errors (including the uncertainty of the empirical distributions) of \pm 0.3 dex. 

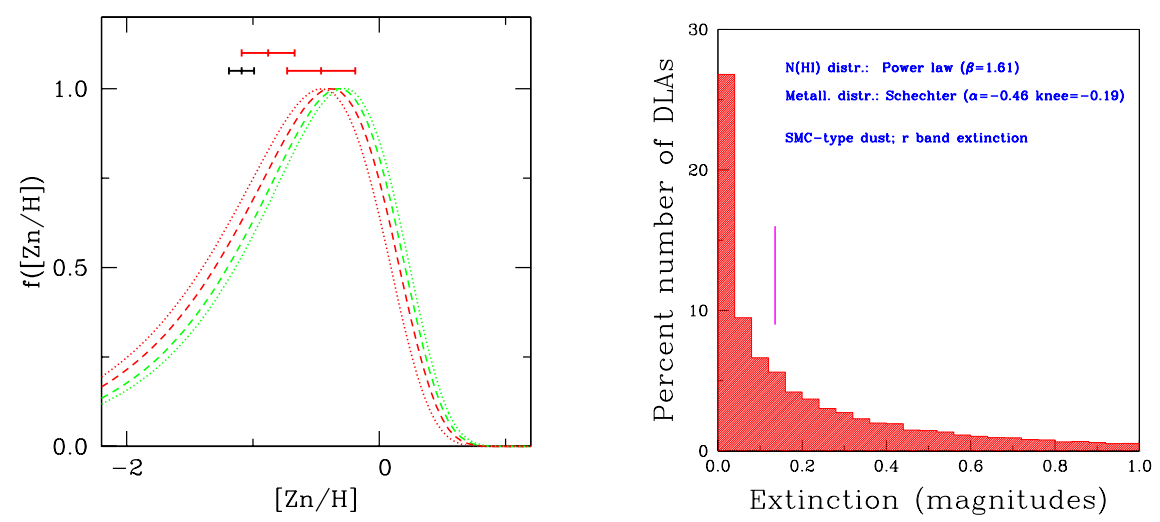

Figure 3. Left: distribution of metallicities in DLAs for different predictions of our models (see Vladilo \& Péroux 2005); the error bars indicate, from left to right, the $\pm 1 \sigma$ errors of the mean metallicity of DLAs estimated (1) at face value, (2) from the CORALS survey (Ellison 2005), and (3) from our predictions. Right: distribution of DLA extinctions in the $r$ band obtained for the ZnII sample of DLAs for an SMC-type dust; vertical bar: median extinction.

The mean metallicity that we derive is higher than the canonical value of DLAs taken at face value, $<[\mathrm{Zn} / \mathrm{H}]>\simeq-1.1$ dex. As we show in Fig. 3, our estimate is consistent at $\approx 1 \sigma$ level with the mean metallicity of the unbiased CORALS sample (Ellison 2005). In the full paper we also show that our predictions of the number density, $n_{\mathrm{DLA}}(z)$, and gas content, $\Omega_{\mathrm{DLA}}$, are consistent with CORALS results (Ellison et al. 2001).

An example of the distribution of DLA extinctions predicted by our study is shown in Fig. 3 (right panel). The distribution has a median value of 0.14 mag in the $r$ band, but is extremely asymmetric. Most DLAs have negligible or little extinction, but the long tail of the distribution, extending up to very high values, indicates that a significant fraction of DLAs may indeed obscure the background quasar. We estimate that the missed fraction is $\approx 30 \%$ to $50 \%$ at limiting magnitude $m_{\ell}=19$, but decreases steadily with $m_{\ell}$. Surveys $m_{\ell} \gtrsim 21$ are weakly affected by the bias.

The extinction distribution highlights the difficulty of measuring the 'mean' DLA reddening using large data sets of quasars. The predicted median reddening is relatively low $(\simeq 0.05$ magnitudes $)$ and hard to detect on the uncertain quasar continuum. In addition, the procedure of stacking a large number of spectra for detecting the 'mean' reddening can give a meaningful answer only if the mean change of continuum slope is a fair representation of the change of slope of individual cases.

The metallicity dependence of the iron dust fraction (Fig. 1, right panel) indicates that DLA extinction should become negligible at $[\mathrm{Zn} / \mathrm{H}] \lesssim-1.5$. In conjunction with the trend of decreasing $[\mathrm{Zn} / \mathrm{H}]$ with redshift (Vladilo et al. 2000), this in turn suggests that DLA extinction may vanish at $z \gtrsim 3$. Therefore, the difficulty of detecting quasar reddening from DLAs at $z \approx 2.8$ (Murphy \& Liske 2004) may be due, in part, to the intrinsic decline of the dust content at high redshift.

An interesting prediction of our method is the fraction of obscured DLAs as a function of $N(\mathrm{ZnII}), \phi\left(N_{\mathrm{Zn}}\right)$. In Fig. 4 (left panel) one can see that $\phi\left(N_{\mathrm{Zn}}\right)$ shows a sharp rise exactly around the Boissé's threshold $\log N(\mathrm{ZnII}) \simeq 13.15$. This remarkable result, obtained without tuning the dust parameters, demonstrates that the obscuration effect provides a natural explanation for the threshold. To discard this explanation one should invoke some unknown mechanism for explaining the lack of DLAs with $\log N(\mathrm{ZnII}) \gtrsim 13.15$ and, in addition, one should tune the dust parameters in such a way to move the threshold at 

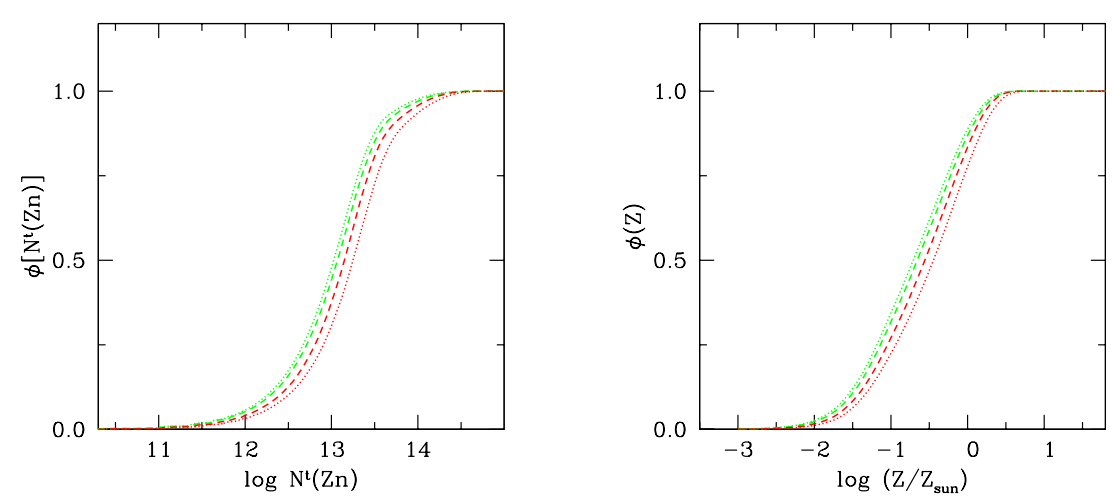

Figure 4. Predicted fraction of obscured DLAs as a function of total $\mathrm{Zn}$ column density (left panel) and metallicity (right panel) in a DLA-quasar survey with limiting magnitude $\simeq 19$ for different models (MW- and SMC-type dust; $r$ and $g$ photometric bands).

much higher values of $\log N(\mathrm{ZnII})$. Our explanation is certainly more attractive in terms of economy of hypothesis.

The reality of Boissé's threshold has been invoked to bring into agreement predictions of galactic chemical evolution models (Prantzos \& Boissier 2000) and of cosmological simulations (Cen et al. 2003) with seemingly discrepant observations of DLAs. The existence of a physical explanation for the threshold favours a scenario in which the predictions of such models are generally correct and the obscuration bias plays an important role.

The combination of the limit $\log N(\mathrm{ZnII}) \leqslant 13.15$ with the cutoff $\log N(\mathrm{HI}) \geqslant 20.3$ imposed in the surveys creates a cutoff of the highest possible metallicity detectable in DLAs. The way the obscuration bias affects the metallicity distribution is shown in the right panel of Fig. 4. One can see that the bias severely hampers the detection of DLAs with near Solar metallicity. Because of the implications for our understanding of the star formation history of the Universe, the effect on the metallicity distribution is probably the most important consequence of the obscuration bias.

\section{References}

Boissé, P., Le Brun, V., Bergeron, J., Deharveng, J. M., 1998, A\&A, 333, 841

Cen, R., Ostriker, J. P., Prochaska, J. X., Wolfe, A., 2003 ApJ, 598, 741

Ellison, S. L., 2005, these proceedings

Ellison, S. L., Yan, L., Hook, I. M., Pettini, M., Wall, J. V., Shaver, P., 2001, A\&A, 379, 393

Fall, S. M., Pei, Y., 1993, ApJ, 402, 479

Khare, P., York, D. G., Vanden Berk, D., et al., 2005, these proceedings

Lopez, S., 2005, these proceedings

Meurer, G. R., 2004, in: A. N. Witt et al. (eds.), Astrophysics of Dust, ASP Conf. Ser., 309, 195

Murphy, M. T., Liske, J., 2004, MNRAS, 354, L31

Ostriker, J. P., Heisler, J., 1984, ApJ, 278, 1

Pei, Y. C., Fall, S. M., Bechtold, J., 1991, ApJ, 378, 6

Prantzos, N., Boissier, S., 2000, MNRAS, 315, 82

Spitzer, L., 1978, Physical Processes in the Interstellar Medium, (New York: Wiley Interscience)

Vladilo, G., 2004, A\&A, 421, 479

Vladilo, G., Bonifacio, P., Centurión, M., Molaro, P., 2000, ApJ, 543, 24

Vladilo, G., Péroux, C., 2005, A\&A, submitted, (astro-ph/0502137) 